\title{
Resveratrol increase myocardial Nrf2 expression in type 2 diabetic rats and alleviate myocardial ischemia/reperfusion injury (MIRI)
}

\author{
Guiping Xu, Xuan Zhao, Juan Fu, Xiaoli Wang \\ Department of Anesthesiology, People's Hospital of Xinjiang Uygur Autonomous Region, Urumqi 830002, China \\ Contributions: (I) Conception and design: G Xu; (II) Administrative support: G Xu; (III) Provision of study materials or patients: X Zhao; (IV) \\ Collection and assembly of data: X Zhao; (V) Data analysis and interpretation: X Zhao; (VI) Manuscript writing: All authors; (VII) Final approval of \\ manuscript: All authors. \\ Correspondence to: Guiping Xu. Department of Anesthesiology, People's Hospital of Xinjiang Uygur Autonomous Region, Urumqi 830002, China. \\ Email: xgpsyl@126.com.
}

Background: Oxidative stress is one of the main factors that increases reperfusion injury in the diabetic heart. Resveratrol could decrease oxidation and promote antioxidant factor expression. Nrf2 is an important endogenous antioxidant. making the heart more resistant to ischemic injury. As Nrf2 expression is considered to be reduced in diabetic heart, we therefore hypothesized that up-regulation of Nrf2 in the diabetic heart may overcome its increased susceptibility to ischemic injury.

Methods: A total of 50 diabetic rats were randomly divided into five groups: the sham operation group $(\mathrm{DM}+$ sham), the MIRI group $(\mathrm{DM}+\mathrm{MI} / \mathrm{R})$, the resveratrol treatment group $(\mathrm{DM}+\mathrm{MI} / \mathrm{R}+\mathrm{RSV})$, the resveratrol + ex527 (SIRT1 inhibitor) group (DM + MI/R + RSV + ex527), and the resveratrol + LY294002 (Akt inhibitor) group (MD + MI/R + RSV + LY294002). Another 20 normal rats were randomly divided into two groups: the sham-operated group $(\mathrm{CON}+$ sham) and the myocardial ischemia-reperfusion group $(\mathrm{CON}+\mathrm{MI} / \mathrm{R})$. A type 2 diabetes model was induced by a high-fat diet-fed and intraperitoneal injection of streptozotocin (STZ). All rats were subjected to $30 \mathrm{~min}$ myocardial ischemia followed by $120 \mathrm{~min}$ reperfusion except sham groups. Plasma were collected to measure the creatine kinase-MB (CK-MB), lactate dehydrogenase (LDH), malondialdehyde (MDA) level, glutathione (GSH) level, and superoxide dismutase (SOD) activity. Pathologic changes in myocardial tissues were observed by Hematoxylin and eosin (HE) straining. SIRT1, p-GSK3 $\beta$, Nrf2, HO-1 protein expressions were measured by western blot.

Results: Compared with the control (CON) group, the diabetic (DM) group had more severe myocardial injury, higher oxidative stress index increase, and a more reduced expression of Nrf2 in the myocardium. After supplementing with resveratrol, the myocardial damage was reduced and the oxidative stress index decreased in the DM group, while the Nrf2 in the myocardium was increased. It also was found that inhibition of SIRT1 also partially inhibited the expression of $\mathrm{Nrf} 2$ and the corresponding antioxidant factor HO-1. Decreased expression of p-GSK3 $\beta$ by Akt inhibitors also partially inhibited Nrf2 and HO-1 expression.

Conclusions: Resveratrol can enhance the expression of Nrf2 in a diabetic heart by stimulating SIRT1 or inhibiting GSK3 $\beta$, alleviating myocardial oxidative stress, and improving ischemia-reperfusion injury.

Keywords: Resveratrol; diabetes; myocardial ischemia/reperfusion; Nrf2; SIRT1; GSK3 $\beta$

Submitted Sep 23, 2019. Accepted for publication Nov 26, 2019.

doi: $10.21037 /$ apm.2019.11.25

View this article at: http://dx.doi.org/10.21037/apm.2019.11.25 


\section{Introduction}

Ischemic heart disease, especially acute myocardial infarction, has become a leading cause of morbidity and disability in Chinese diabetes (1). After reperfusion therapy, diabetes combined with acute myocardial infarction often leads to more severe reperfusion injury. Therefore, the sensitivity and prognosis of a diabetic heart to reperfusion injury is worse than in the nondiabetic population. Meanwhile, diabetes can also eliminate the protective effects of various drugs or ischemic preconditioning on the ischemic myocardium (2,3). Accumulated studies have demonstrated that hyperglycemia aggravating oxidative stress is one of the main factors that increases reperfusion injury in the diabetic heart (4). Therefore, enhancing the depletion of antioxidants in patients with diabetes is the key to protecting the heart against myocardial ischemia reperfusion injury (MIRI).

Resveratrol, a natural polyphenolic anti-toxin present in several plants including grapes, has been widely shown to have potent antioxidant properties $(5,6)$. Resveratrol could decrease oxidation and promote antioxidant factor expression (7). A large number of studies have shown that resveratrol could help in protecting against cardiovascular diseases, especially MIRI (8), through anti-oxidative stress, anti-inflammatory, anti-apoptosis, and other effects $(9,10)$, Recently, resveratrol has been shown to enhance the antioxidant activity of the diabetic heart, by reducing fasting blood glucose to increase insulin sensitivity (11). However, whether resveratrol can alleviate diabetic MIRI and exert a protective molecular effect remains unknown.

Various intracellular signaling pathways were proven to participate in resveratrol's antioxidant actions, including Nrf2/ARE signaling pathway, silent information regulator 1 (SIRT1), PI3K/Akt/GSK3 $\beta$ signaling pathway, nitric oxide-synthase (NOS), adenosine 5'-monophosphate (AMP)-activated protein kinase (AMPK), etc. (12-14). Resveratrol is considered to be an indirect Nrf2 agonist. $\mathrm{Nrf2}$, a transcription factor, is an important endogenous antioxidant. Under certain physiological conditions, Nrf2 interacts with Kelch-like ECH-associated protein 1 (Keap1) in the cytoplasm, which facilitates Nrf2 ubiquitination and degradation. Upon exposure of MIRI, excessive production of oxygen-free radicals, known as oxidative stress, occurs, and Nrf2 is activated. Nrf2 then splits from Keap 1 and translocates into nucleus, enhancing the expression of downstream antioxidant factors such as HO-1 (15). However, studies have shown that the expression of $\mathrm{Nrf2}$ is lower in diabetic patients and animal models (16-18), suggesting that the inhibition of $\mathrm{Nrf2}$ may be related to the increased reperfusion injury of a diabetic heart. The activation of Nrf2 involves a variety of signaling pathways. Studies have shown that the activation of SIRT1 to promote Nrf2-ARE anti-oxidative pathway can resist the excessive oxidative stress caused by a high glucose environment (19), while the inhibition of GSK3 $\beta$ to enhance the expression of Nrf2 and HO-1 has been shown to reduce MIRI (20). GSK3 $\beta$ and SIRT1 are related factors of diabetic cardiovascular complications. Activation of SIRT1-PCG-1 signaling pathway can reduce oxidative stress and inflammatory response in the diabetic heart, alleviate myocardial fibrosis, and improve cardiac function in diabetes (21); inhibition of GSK3 $\beta$ can maintain the mitochondrial integrity of the diabetic heart and increase the survival of diabetic cardiomyocytes (22). Therefore, we used resveratrol to change the expression of GSK-3 $\beta$ and SIRT1 and observed whether this could restore the expression of $\mathrm{Nrf} 2$ in a diabetic heart through a protective effect.

Accordingly, this study established a model of type 2 diabetes and a model of diabetic MIRI, and was designed with the following objectives: (I) identify the mechanism underlying the type 2 diabetic state aggravating MIRI with Nrf2 signaling pathway; (II) explore the protective effect of resveratrol supplement on MIRI in a type 2 diabetic model; (III) investigate whether activation of SIRT1 and inhibition of GSK3 $\beta$ can restore the expression of $\mathrm{Nrf} 2$ in diabetic heart, and explore the relative role of SIRT1, GSK3 $\beta$, and Nrf2 in diabetic MIRI.

\section{Methods}

\section{Animals}

Healthy male Sprague-Dawley (SD) rats weighing 220-280 g were supplied by the Experimental Animal Center of the First Affiliated Hospital of Xinjiang Medical University. All experimental rats were conditioned for 2 weeks, alternated for 12 hours, and were housed at a room temperature of $25-28{ }^{\circ} \mathrm{C}$ and $60 \%$ humidity. The experimental protocols were approved by the Experimental Animal Ethics Committee.

\section{Experimental groups}

All rats were randomly divided into a control group (CON, 
$\mathrm{n}=20)$ and a diabetic group (DM, $\mathrm{n}=50)$. Rats in the control group were further divided randomly into 2 groups $(n=10)$ : the sham-operated group (CON + sham) and the myocardial ischemia-reperfusion group (CON + MI/R). Rats in the diabetic group were further randomized into 5 groups $(n=10)$ : the sham operation group (DM + sham), the MIRI group $(\mathrm{DM}+\mathrm{MI} / \mathrm{R})$, the resveratrol treatment group $(\mathrm{DM}+\mathrm{MI} / \mathrm{R}$ + RSV), the resveratrol + ex527 (SIRT1 inhibitor) group (DM $+\mathrm{MI} / \mathrm{R}+\mathrm{RSV}+\mathrm{ex} 527)$, and the resveratrol + LY294002 (Akt inhibitor) group (MD + MI/R + RSV + LY294002). Resveratrol was injected intraperitoneally $20 \mathrm{mg} / \mathrm{kg}$ for 7 days (23); the SIRT1 inhibitor ex527 $1 \mu \mathrm{g} / \mathrm{kg}$ was given as intravenous injections $15 \mathrm{~min}$ before ischemia (24); LY294002 $1.5 \mathrm{mg} / \mathrm{Kg}$ was given as intravenous injections $30 \mathrm{~min}$ before ischemia (25), and other groups were separately injected with normal volumes of saline.

\section{Type 2 diabetic model}

A type 2 diabetes model was induced by a high-fat diet-fed and intraperitoneal injection of streptozotocin (STZ) (26). After 2 weeks of adaptive feeding, the rats were fed the high-fat diet containing $40 \%$ fat, $41 \%$ carbohydrate, and $18 \%$ protein for 6 weeks. After that, STZ (Sigma, USA) $35 \mathrm{mg} / \mathrm{Kg}$ was intraperitoneally injected. The rats were then continuously fed the high-fat diet. Rats presented typical symptoms of diabetes and showed a blood glucose level of above $>16.7 \mathrm{mmol} / \mathrm{L}$ at least 3 times, thus meeting the criteria for a diabetes diagnosis.

\section{Myocardial infarction protocol}

We used a well-established model of MI/R injury (27). Before the surgery, all experimental animals were anesthetized, and the anterior descending branch of the left coronary artery was occluded for $30 \mathrm{~min}$ followed by reperfusion for $120 \mathrm{~min}$. Ischemia was verified by the elevation of ST-T segment with limb lead II. After $30 \mathrm{~min}$ of ischemia, the ligature was loosened. The myocardial color became red and the ST-T segment returned to the pre-ischemic level, suggesting that reperfusion was successful. At the end of reperfusion, blood and tissue samples were collected for further analysis. The sham operation group underwent the same surgical procedure except that the suture passed under the anterior descending branch of the left coronary artery.

\section{Hematoxylin and eosin (HE) straining}

Myocardial tissues were embedded in paraffin and prepared for sectioning. They were fixed in $4 \%$ paraformaldehyde solution for $24 \mathrm{~h}$, stained with $\mathrm{HE}$, and pathological results were observed under a light microscope (×200).

\section{Determination of myocardial infarction}

At the end of $120 \mathrm{~min}$ of reperfusion, myocardial infarction was determined by means of a double-staining technique and a digital imaging system (infarct area/area at risk $\times 100 \%$ ).

\section{Measurement of creatine kinase-MB (CK-MB) and lactate debydrogenase (LDH)}

Blood samples $(2 \mathrm{~mL})$ were drawn at $120 \mathrm{~min}$ after reperfusion. Serum CK-MB and LDH were measured spectrophotometry (Elabscience, Wuhan, China).

\section{Measurement of malondialdehyde (MDA) level, glutathione (GSH) level, superoxide dismutase (SOD) activity}

A myocardial tissue sample was taken after $120 \mathrm{~min}$ of reperfusion and tested for malondialdehyde MDA content, GSH content, and SOD activity according to the test kit instructions (Nanjing Kaiji Bio, Nanjing, China).

\section{Western blotting}

Total protein was separated by SDS-PAGE gel and then transferred to a polyvinylidene difluoride (PVDF) membrane. After blocking the PVDF membranes with 5\% fat-free milk for $2 \mathrm{~h}$, proteins were respectively probed with a specific antibody [anti-SIRT1 (1:1,000), anti-pGSK3 $\beta$ $(1: 1,000)$, anti-Nrf2 $(1: 1,000)$, anti-HO-1 $(1: 1,000)$, anti- $\beta$ actin (SantaCruz Biotechnology)] and incubated overnight. After washing 3 times with TBST buffer, the complexes were detected by secondary antibodies. The bands were quantified by scanning densitometry using a Bio-Image Analysis System (Bio-Rad, Richmond, CA, USA).

\section{Statistical analysis}

All data were presented as means + S.E.M. Differences were compared by analysis of variance (ANOVA) followed by Bonferroni correction for post hoc t-test, where appropriate. Probabilities of $<0.05$ were considered to be statistically significant. All of the statistical analyses were performed with the GraphPad Prism software version 7.0 (GraphPad Software, San Diego, CA, USA). 


\section{Results}

As shown in Table 1, after STZ + high-fat-diet induced diabetes, the type 2 diabetic rats showed characteristic symptoms of diabetes including hyperglycemia, polydipsia, and polyphagia. The body weights of diabetic rats were also lower, and this fact, in combination with the above data, indicated that the type 2 diabetic animals were successfully developed.

As shown in Figure 1, myocardial HE staining showed a

Table 1 Characteristics of control and diabetic rats

\begin{tabular}{lrc}
\hline Basic parameters & CON & DM \\
\hline Water intake (mL/kg per day) & $121 \pm 9.40$ & $904 \pm 70.27^{* *}$ \\
Food consumption (g/kg per day) & $61.8 \pm 4.54$ & $193 \pm 10.12^{\star *}$ \\
Fasting blood glucose (mmol/L) & $5.5 \pm 0.46$ & $23.84 \pm 1.76^{\star *}$ \\
Body weight (g) & $471.2 \pm 29.14$ & $236.4 \pm 11.19^{* *}$ \\
\hline
\end{tabular}

Results are expressed as means $\pm S D$ for $n=10$. Compared with control group, ${ }^{\star \star} \mathrm{P}<0.01$. CON, control; DM, diabetes mellitus. small amount of inflammatory cell infiltration in the DM + sham group compared with the CON + sham group; significant necrosis and inflammatory cell infiltration were observed in the $\mathrm{DM}+\mathrm{MI} / \mathrm{R}$ group compared with the $\mathrm{CON}+\mathrm{MI} / \mathrm{R}$ group. In addition, compared with the sham group, the myocardial infarct volume increased in the MI/R group $(\mathrm{P}<0.05$, Figure $2 A)$. However, the myocardial infarction volume in the $\mathrm{DM}+\mathrm{MI} / \mathrm{R}$ group was further increased (compared with the $\mathrm{CON}+\mathrm{MI} / \mathrm{R}$ group, $\mathrm{P}<0.05$ ); consistently, the myocardial injury indexes of LDH and CK$M B$ were further increased in the $\mathrm{DM} M \mathrm{MI} / \mathrm{R}$ group (compared with the $\mathrm{CON}+\mathrm{MI} / \mathrm{R}$ group, $\mathrm{P}<0.05$, Figure $2 B, C)$. These results suggest that diabetes further aggravated myocardial damage in the rats subjected to $M I / R$ injury.

As shown in Figure $3 A, B, C, \mathrm{MI} / \mathrm{R}$ injured rats showed increased myocardial MDA level and decreased SOD activity and GSH levels in nondiabetic rats (compared with the $\mathrm{CON}+$ sham group, $\mathrm{P}<0.05$ ), while the MDA level was further increased and SOD activity and GSH levels were decreased in the $\mathrm{DM}+\mathrm{MI} / \mathrm{R}$ group. These data indicate that diabetes aggravated myocardial oxidative stress in rats

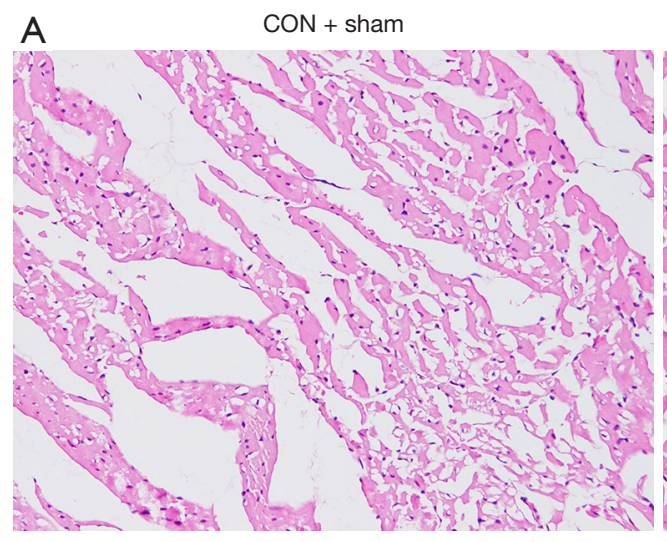

B $\quad \mathrm{CON}+\mathrm{MI} / \mathrm{R}$
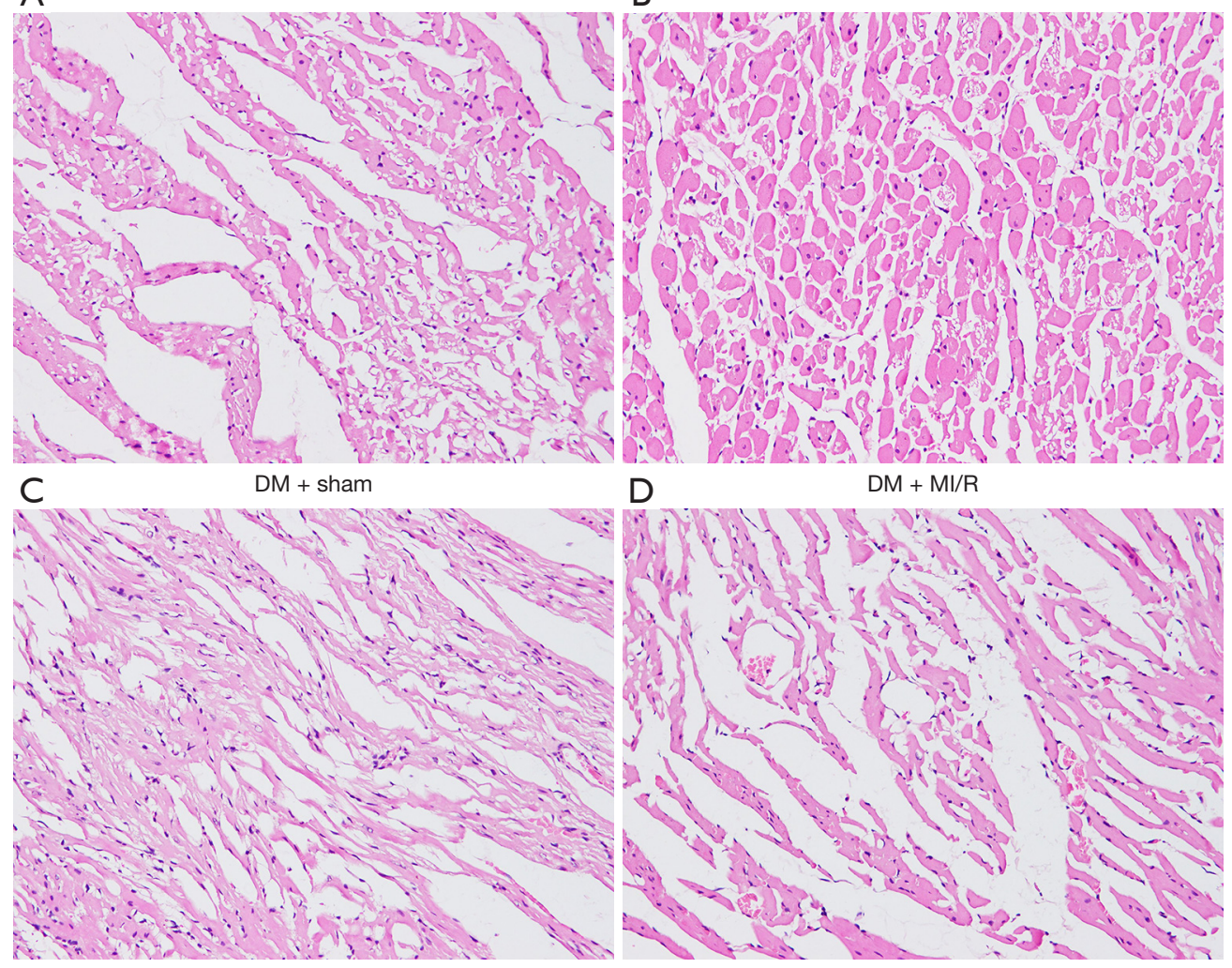

D $\quad \mathrm{DM}+\mathrm{MI} / \mathrm{R}$

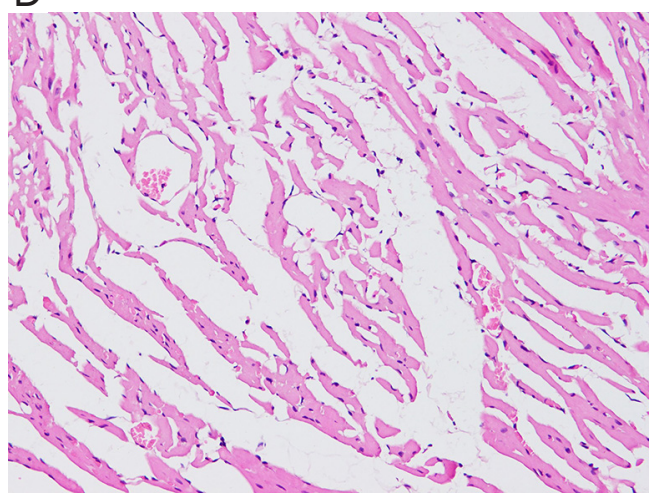

Figure 1 Light microscopic observation of myocardial tissue section HE staining pathology ( $\times 200)$. 

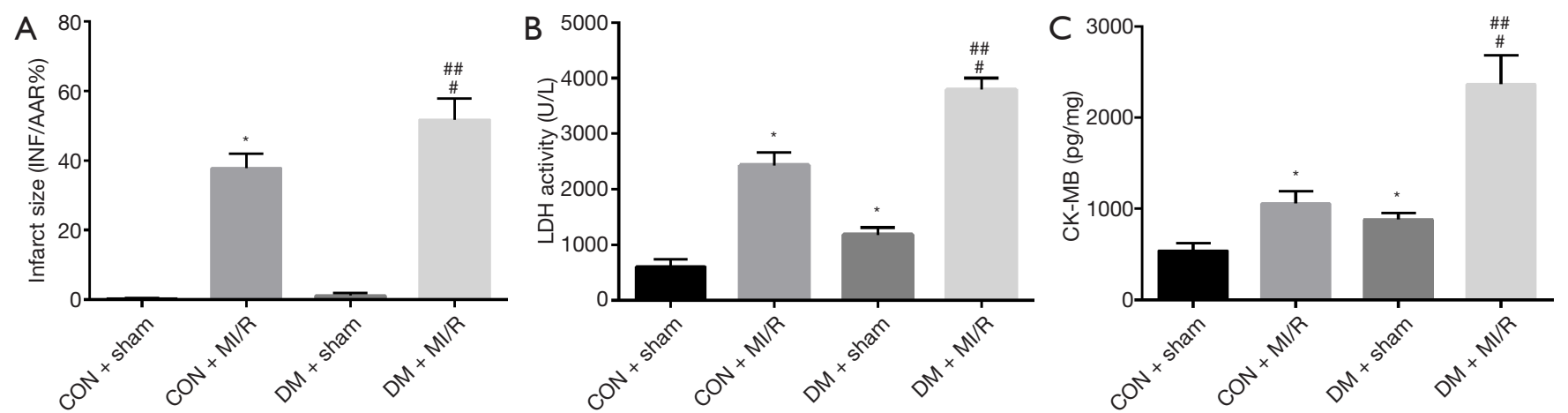

Figure 2 Comparison of myocardial infarct volume and myocardial injury index in normal and diabetic rats. (A) Infarct size; (B) LDH; (C) CK-MB. Compared with $\mathrm{CON}+$ sham group, ${ }^{*} \mathrm{P}<0.05$; compared with $\mathrm{DM}+$ sham group, ${ }^{\#} \mathrm{P}<0.05$; compared with $\mathrm{CON}+\mathrm{MI} / \mathrm{R}$ group, ${ }^{\#} \mathrm{P}<0.05$. LDH, lactate dehydrogenase; CK-MB, creatine kinase-MB.
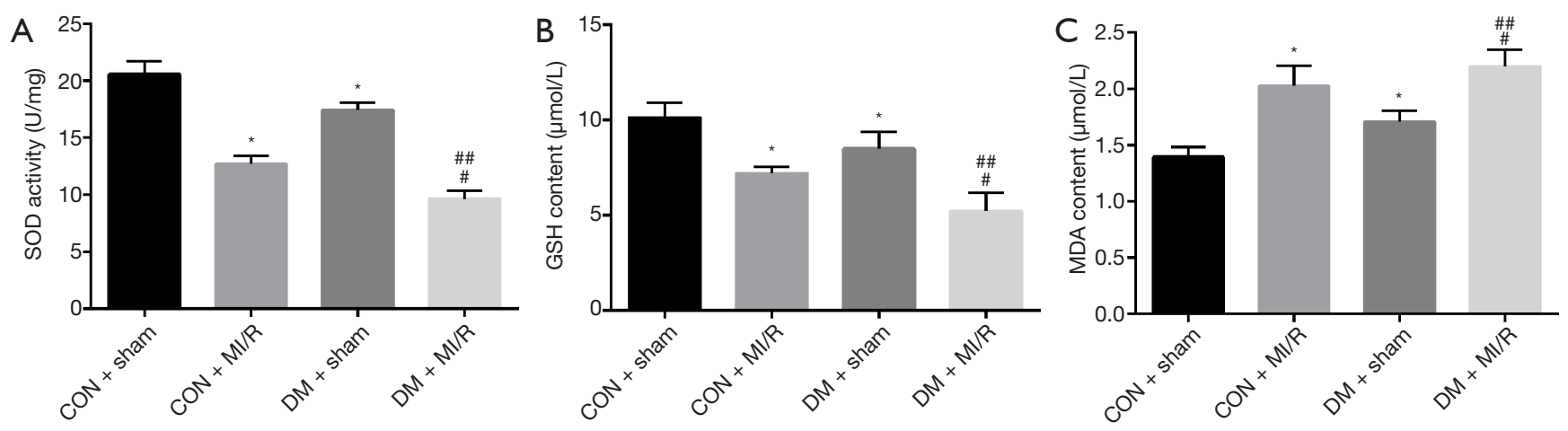

Figure 3 Comparison of oxidative stress indicators in normal and diabetic rats. (A) SOD; (B) GSH; (C) MDA. Compared with CON + sham group, ${ }^{*} \mathrm{P}<0.05$; compared with $\mathrm{DM}+$ sham group, ${ }^{*} \mathrm{P}<0.05$; compared with the $\mathrm{CON}+\mathrm{MI} / \mathrm{R}$ group, ${ }^{\# \#} \mathrm{P}<0.05$. SOD, superoxide dismutase; GSH, glutathione; MDA, malondialdehyde.

subjected to MIRI.

We next investigated the changes in the expression of Nrf2 and related downstream factors in both diabetic and non-diabetic rats. As shown in Figure 4A,B, the expression of $\mathrm{Nrf} 2$ and its downstream factor, HO-1, were decreased in the $\mathrm{DM}+$ sham group (compared with the CON + sham group, $\mathrm{P}<0.05$ ), indicating myocardial Nrf2 signaling and HO-1 were impaired. In addition, the expression of $\mathrm{Nrf} 2$ and $\mathrm{HO}-1$ in the $\mathrm{DM}+\mathrm{MI} / \mathrm{R}$ group was further reduced (compared with the $\mathrm{CON}+\mathrm{MI} / \mathrm{R}$ group, $\mathrm{P}<0.05)$. These data suggest that myocardial Nrf2 signaling was impaired in the diabetic setting, which led to further aggravation of diabetic MIRI.

We further evaluated the effects of resveratrol on MI/ $\mathrm{R}$ injury in type 2 diabetic rats. As shown in Figure 5, compared with the DM + MI/R group, degree of myocardial damage lighter than in the $\mathrm{DM}+\mathrm{MI} / \mathrm{R}+\mathrm{RSV}$ group. In addition, the myocardial infarction volume was decreased, and the myocardial damage indicators LDH and CK-MB were further reduced (compared with the $\mathrm{DM}+\mathrm{MI} / \mathrm{R}$ group, $\mathrm{P}<0.05$, Figure $6 A, B, C)$. At the same time, in the $\mathrm{DM}$ $+\mathrm{MI} / \mathrm{R}+\mathrm{RSV}$ group, the MDA level was decreased, both GSH content and SOD activity were increased, and the expression of $\mathrm{Nrf} 2$ was increased (compared with the DM $+\mathrm{MI} / \mathrm{R}$ group, $\mathrm{P}<0.05$, Figure $6 D, E, F, G)$. These combined data suggest that resveratrol reduced the oxidative stress in diabetic rats by restoring the expression of $\mathrm{Nrf2}$, and protected the MI/R injury in type 2 diabetic rats.

In present study, we further determined the relationship between SIRT1, GSK3 $\beta$, and Nrf2 after MIRI in type 2 diabetic rats. The expression of SIRT1, p-GSK-3 $\beta$ and HO-1 were significantly increased in the $\mathrm{DM}+\mathrm{MI} / \mathrm{R}+$ $\mathrm{RSV}$ group (compared with the $\mathrm{DM}+\mathrm{MI} / \mathrm{R}$ group, $\mathrm{P}<0.05$, Figure $7 A, B, C, D)$. After the application of the inhibitor, the expression of SIRT1 and p-GSK-3 $\beta$ was respectively 

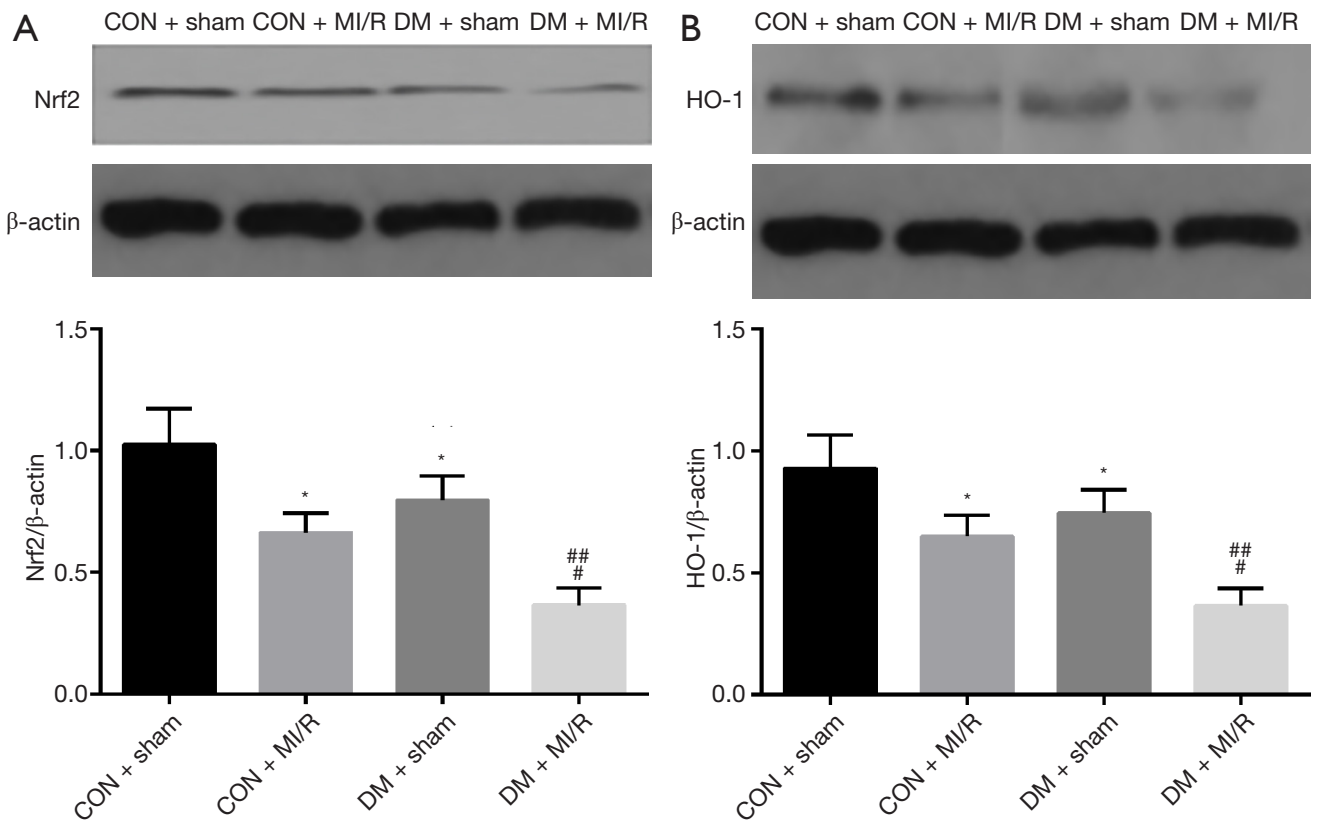

Figure 4 Comparison of Nrf2 and HO-1 expression between the control group and diabetic group. (A) Nrf2; (B) HO-1. Compared with the $\mathrm{CON}+$ sham group, ${ }^{*} \mathrm{P}<0.05$; compared with the $\mathrm{DM}+$ sham group, ${ }^{\#} \mathrm{P}<0.05$; compared with the $\mathrm{CON}+\mathrm{MI} / \mathrm{R}$ group, ${ }^{\#} \mathrm{P}<0.05$. Nrf2, NF-E2-related factor 2; HO-1, heme oxygenase-1.

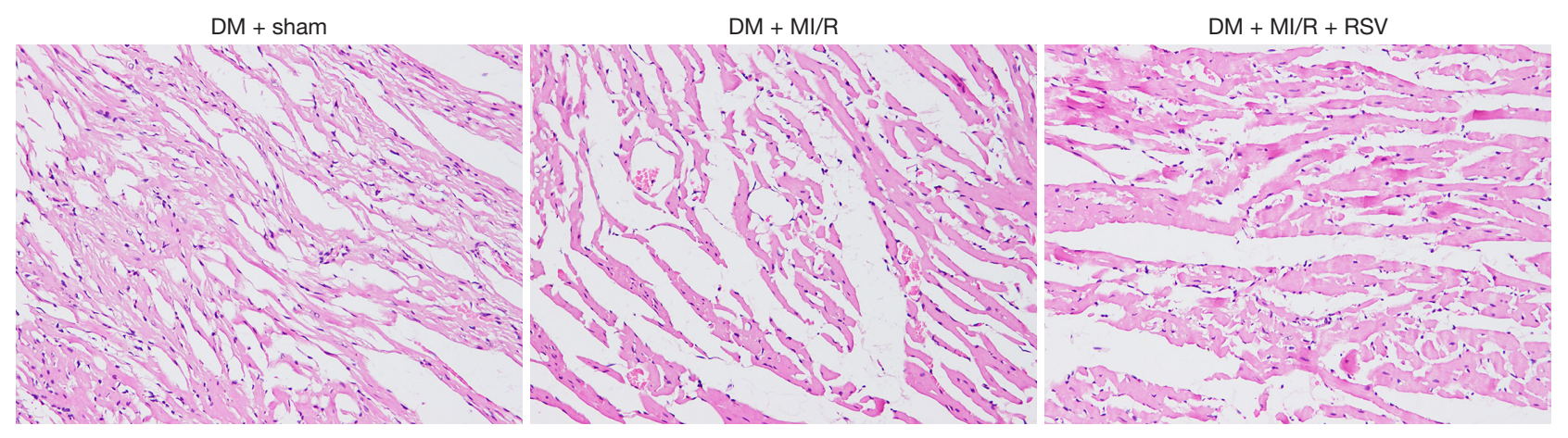

Figure 5 Light microscopic observation of a myocardial tissue section with HE staining pathology $(\times 200)$.

decreased in the $\mathrm{DM}+\mathrm{MI} / \mathrm{R}+\mathrm{RSV}+$ ex527 group, and, in the $\mathrm{DM}+\mathrm{MI} / \mathrm{R}+\mathrm{RSV}+\mathrm{LY} 294002$ group, the expression of Nrf2 and HO-1 was decreased (compared with the $\mathrm{DM}+\mathrm{MI} / \mathrm{R}+\mathrm{RSV}$ group, $\mathrm{P}<0.05$, Figure $7 A, B)$. However, the differences in the expression of $\mathrm{Nrf2}$ and HO-1 were slightly lower in the DM + MI/R + RSV + ex527 group, but there was no statistically significant difference between the $\mathrm{DM}+\mathrm{MI} / \mathrm{R}+\mathrm{RSV}$ group and the $\mathrm{DM}+\mathrm{MI} / \mathrm{R}+\mathrm{RSV}+$ ex527 group, $(\mathrm{P}>0.05$, Figure $7 C, D)$. In the $\mathrm{DM}+\mathrm{MI} / \mathrm{R}+$ RSV + LY294002 group, the expression of Nrf2 and HO-1 were reversed after the application of the inhibitor $(\mathrm{P}<0.05$,
Figure $7 C, D)$. These changes, taken as a whole, suggest that SIRT1 and GSK3 $\beta$ together regulate the expression of Nrf2 in a diabetic heart and participate in the protective effect of resveratrol.

\section{Discussion}

In the present study, it was found that hyperglycemia aggregates oxidative stress and is associated with the impairment of Nrf2 signaling pathway, which may be one of the main factors that aggravates MIRI. Resveratrol 

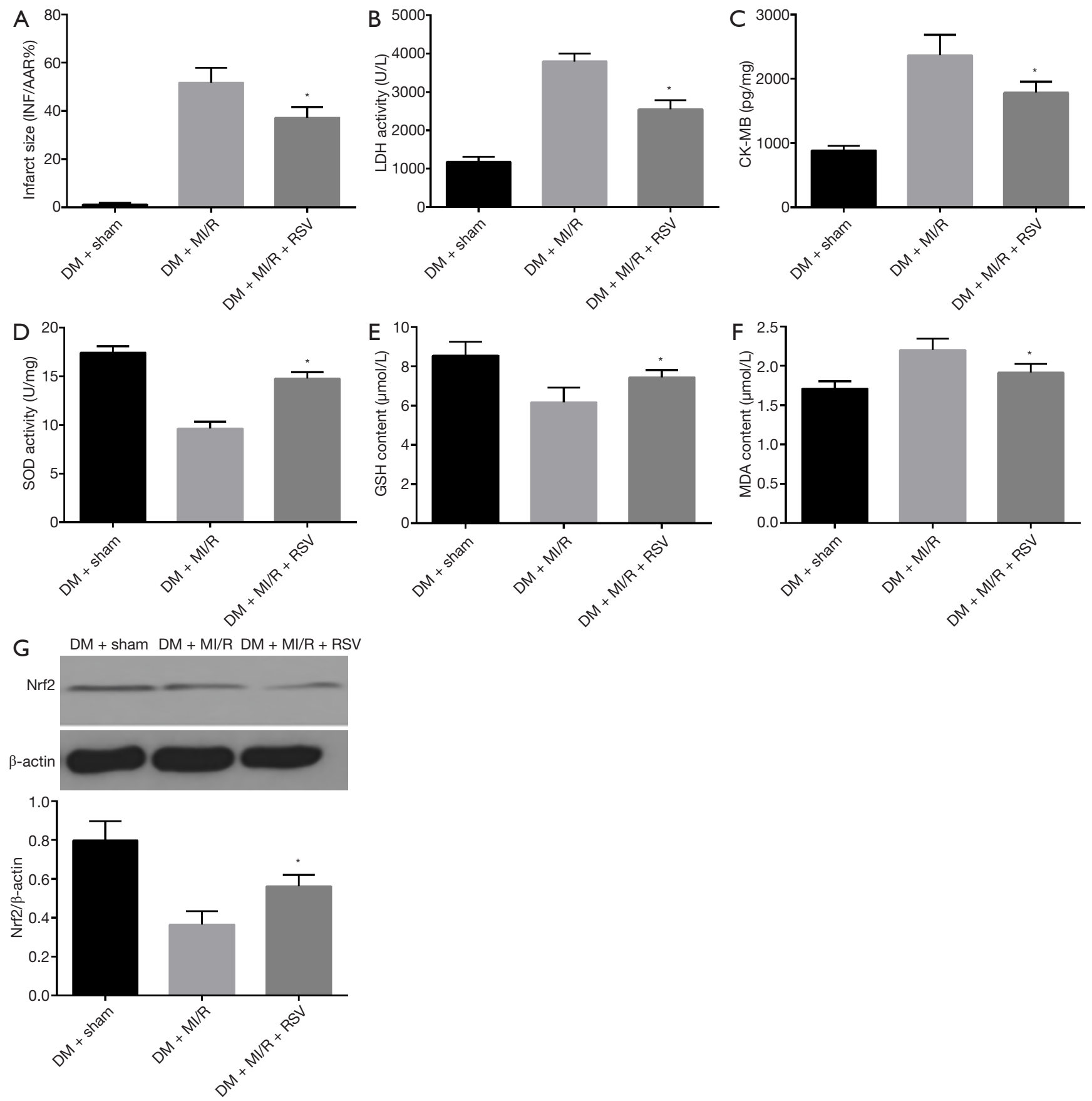

Figure 6 Protective effect of resveratrol on MIRI in diabetic rats. (A) Infarct size; (B) LDH; (C) CK-MB; (D) SOD; (E) GSH; (F) MDA; (G) Nrf2. Compared with the DM + MI/R group, ${ }^{*} \mathrm{P}<0.05$. LDH, lactate dehydrogenase; CK-MB, creatine kinase-MB; SOD, superoxide dismutase; GSH, glutathione; MDA, malondialdehyde; Nrf2, NF-E2-related factor 2.

upregulates SIRT1 and downregulates GSK3 $\beta$, contributing to improving the expression of $\mathrm{Nrf2}$, inhibiting oxidative stress, and attenuating diabetic MI/R injury.

Resveratrol has attracted substantial attention by virtue of its antioxidant properties. A large number of studies have demonstrated the antioxidant properties of resveratrol which include reducing the production of malondialdehyde (MDA) and restoring glutathione (GSH) 

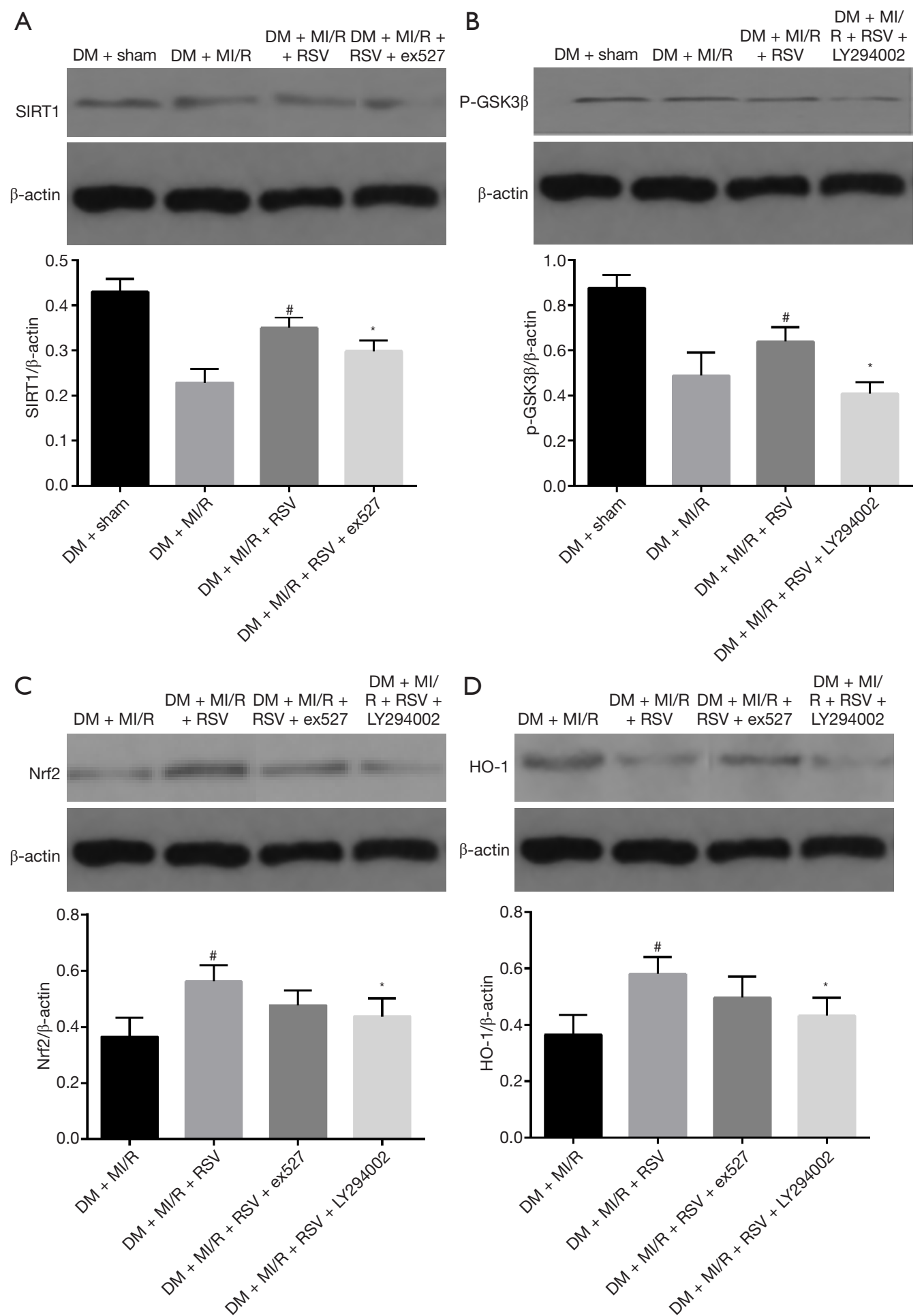

Figure 7 To evaluate the relationship between SIRT1 and GSK3 $\beta$ in reversing MIRI and Nrf2 in rats with type 2 diabetes mellitus. (A) SIRT1; (B) p-GSK3ß; (C) Nrf2; (D) HO-1. Compared with the DM + MI/R group, ${ }^{\text {PP }}<0.05$; compared with the DM + MI/R + RSV group, ${ }^{*}<<0.05$. SIRT1, silent information regulator 1 ; p-GSK3 $\beta$, glycogen synthase kinase-3 $\beta$; Nrf2, NF-E2-related factor 2; HO-1, heme oxygenase-1. 
levels to improve neurological function. Resveratrol also inhibits oxidative stress through the SIRT1/FOXO3a pathway to reduce tubular damage caused by hyperglycemia $(28,29)$. Therefore, resveratrol is used in the treatment of diseases related to oxidative stress, such as ischemiareperfusion injury. It is also worth noting that resveratrol has a protective effect on MIRI by enhancing the expression of Nrf2 (30,31). Resveratrol has also been found to possess the effect of reducing insulin resistance and lowering plasma glucose level. Therefore, in recent years, resveratrol has also been studied for the treatment of diabetes and its related complications. Because type 2 diabetes often nullifies the effects of multiple protective factors on MIRI, it is still unclear whether resveratrol has protective effects on diabetic MIRI. Through a consideration of previous experimental data and the findings of the present study, we can conclude that resveratrol not only has protective effects on myocardial ischemia-reperfusion injury in normal rats, but also has a protective effect on myocardial ischemiareperfusion injury in type 2 diabetic rats, with its protective mechanism potentially being related to enhancing the expression of $\mathrm{Nrf} 2$ and further reducing the oxidative stress of the diabetic heart. Furthermore, our study found that the expression of $\mathrm{Nrf} 2$ in the diabetic heart was impaired, and that a resveratrol supplement restored Nrf2 expression and protected the diabetic heart.

A growing number of experimental studies have illustrated that Nrf2 can attenuate ischemia-reperfusion injury and effectuate cardioprotective effects. This endogenous protective effect ultimately inhibits the production of oxygenfree radicals and reduces oxidative stress by promoting the expression of antioxidant factors such as HO- 1 . The present experiment demonstrated that the expression of Nrf2 was reduced in the diabetic heart, which was consistent with the conclusion of Tan (17). This result suggests that impaired expression of $\mathrm{Nrf} 2$ induces oxidative stress in the hyperglycemic environment. Currently, it is believed that hyperglycemia caused by type 2 diabetes is mainly caused by insulin resistance. We thus inferred that the down-regulation of $\mathrm{Nrf} 2$ expression in the diabetic heart aggravates oxidative stress and insulin resistance, resulting in myocardial damage. However, Peak et al. (32) have the opposing view: they assert that the expression of Nrf2 in the diabetic heart is increased. We believe that this discrepancy of experimental results can be accounted for by the differences of the duration of diabetes status, animal species, and experimental protocols between the studies.

In this study, in order to confirm whether the recovery of expression of $\mathrm{Nrf2}$ in a diabetic heart can alleviate MIRI in diabetic rats, we used resveratrol to restore the expression of Nrf2 in the diabetic heart, and explore whether improving the expression of Nrf2 in a diabetic heart could reduce the MIRI. We explored the effect of resveratrol on MIRI in diabetic heart in vivo experiments. By intraperitoneal injection of resveratrol, this study found that compared with the $\mathrm{DM}+\mathrm{MI} / \mathrm{R}$ group, the expression of $\mathrm{Nrf} 2$ in the $\mathrm{DM}$ $+\mathrm{RSV}+\mathrm{MI} / \mathrm{R}$ group increased, the myocardial infarction volume decreased, and the myocardial injury index decreased accordingly. This experiment also demonstrated that the application of resveratrol can enhance the expression of $\mathrm{Nrf2}$ and reduce the oxidative stress of the diabetic heart. It is worth noting that resveratrol does not act as a direct agonist of Nrf2, and the fact that that resveratrol enhances Nrf2 expression in the diabetic heart may also suggest that it may be involved in the regulation of certain upstream factors.

SIRT1 not only participates in alleviating metabolic disorders and other related diseases, but also protects against oxidative stress in the cardiovascular system. Numerous studies have confirmed the protective effect of SIRT1 agonists on MI/R injury in diabetes $(33,34)$. Resveratrol is often used as a direct agonist of SIRT1 to study the relevant protective mechanisms. In this experiment, we not only observed that SIRT1 signaling was impaired in the diabetic heart, but also found that reperfusion injury led to the further down-regulation of the expression of SIRT1. Resveratrol has been administered to increase the expression of SIRT1, and this change was consistent with the change of $\mathrm{Nrf} 2$ in the diabetic heart; further application of SIRT1 inhibitor ex527 revealed that SIRT1 and Nrf2 were decreased in the diabetic heart, thus confirming that SIRT1 may promote the expression of $\mathrm{Nrf} 2$ in the diabetic heart. Research has shown that SIRT1 regulates Nrf2 to then regulate transcriptional coding of antioxidant genes and thus affect cellular redox status (35); therefore, we can conclude that SIRT1 improves the expression of Nrf2 in the diabetic heart, and that resveratrol further stimulates SIRT1 to promote the expression of $\mathrm{Nrf} 2$ and reduce $\mathrm{MI} / \mathrm{R}$ injury in diabetic rats.

Glycogen synthase kinase-3 $\beta$ (GSK3 $\beta$ ) is a multifunctional serine/threonine kinase that regulates several important cellular signaling pathways including PI3K/Akt (36). When GSK3 $\beta$ is rendered inactive, this inhibits the opening of mPTP and ultimately reduces the production of oxygenfree radicals (37). Indeed, studies have shown that inhibition of GSK $3 \beta$ can reduce MI/R injury (38). GSK3 $\beta$ is also involved in the regulation of insulin signaling, and inhibition of GSK3 $\beta$ activity is considered to have potential anti- 
diabetic effects. In a variety of rodent insulin-resistance models, GSK3 $\beta$ in tissues were found to be highly active (39). Therefore, we aimed to determine whether plays a role in diabetic MI/R injury by changing its expression. In this experiment, Akt inhibitor was used to inhibit the expression of p-GSK3 $\beta$; we found that the expression of Nrf2 was decreased, and the oxidative stress index was increased. However, the expression of p-GSK3 $\beta$ was more increased after resveratrol administration, compared with the MI/ $\mathrm{R}$ group. Therefore, the expression of Nrf2 may also be affected by GSK3 3 .

In conclusion, our experimental data demonstrates the protective effect of resveratrol on reperfusion injury in the diabetic ischemic heart. Resveratrol restores the expression of Nrf2 in the type 2 diabetic heart, which is related to a reduction in I/R injury. In addition, we also found that up-regulating SIRT1 and down-regulating GSK3 $\beta$ in the diabetic heart ultimately activated Nrf2 signal pathway. Improving the expression of $\mathrm{Nrf} 2$ may be a viable strategy to reduce MIRI in diabetes. However, we mainly arrived at the above conclusions based on in vivo experiments. In order to further explore the specific molecular regulation mechanism, it is necessary to carry out further vitro experiments.

\section{Acknowledgments}

None.

\section{Footnote}

Conflicts of Interest: The authors have no conflicts of interest to declare.

Ethical Statement: The authors are accountable for all aspects of the work in ensuring that questions related to the accuracy or integrity of any part of the work are appropriately investigated and resolved. The experimental protocols were approved by the Experimental Animal Ethics Committee (IACUC20170420-02).

\section{References}

1. Bragg F, Holmes MV, Iona A, et al. Association Between Diabetes and Cause-Specific Mortality in Rural and Urban Areas of China. JAMA 2017;317:280-9.

2. Jiang F, Gu EW, Lu XF, et al. Effect of type 2 diabetes mellitus on attenuation of myocardial ischemia-reperfusion injury by sufentanil postconditioning in rats. Chin J
Anesthesiol 2013;33:228-31.

3. Saeid F, Aniseh J, Reza B, et al. Signaling mediators modulated by cardioprotective interventions in healthy and diabetic myocardium with ischaemia-reperfusion injury. Eur J Prev Cardiol 2018;25:1463-81.

4. Lejay A, Fang F, John R, et al. Ischemia reperfusion injury, ischemic conditioning and diabetes mellitus. J Mol Cell Cardiol 2016;91:11-22.

5. Averilla JN, Oh J, Wu Z, et al. Improved extraction of resveratrol and antioxidants from grape peel using heat and enzymatic treatments. J Sci Food Agric 2019;99:4043-53.

6. Li YR, Li S, Lin CC. Effect of resveratrol and pterostilbene on aging and longevity. Biofactors 2018;44:69-82.

7. Cottart CH, Nivet-Antoine V, Beaudeux JL. Review of recent data on the metabolism, biological effects, and toxicity of resveratrol in humans. Mol Nutr Food Res 2014;58:7-21.

8. Li J, Xie C, Zhuang J, et al. Resveratrol attenuates inflammation in the rat heart subjected to ischemiareperfusion: Role of the TLR4/NF- $\mathrm{kB}$ signaling pathway. Mol Med Rep 2015;11:1120-6.

9. Mokni M, Hamlaoui S, Karkouch I, et al. Resveratrol Provides Cardioprotection after Ischemia/reperfusion Injury via Modulation of Antioxidant Enzyme Activities. Iran J Pharm Res 2013;12:867-75.

10. Yang $L$, Zhang $Y, Z$ hu $M$, et al. Resveratrol attenuates myocardial ischemia/reperfusion injury through upregulation of vascular endothelial growth factor B. Free Radic Biol Med 2016;101:1-9.

11. Bagul PK, Middela H, Matapally S, et al. Attenuation of insulin resistance, metabolic syndrome and hepatic oxidative stress by resveratrol in fructose-fed rats. Pharmacol Res 2012;66:260-8.

12. Bonnefont-Rousselot D. Resveratrol and Cardiovascular Diseases. Nutrients 2016;8:250.

13. Li H, Xia N. Cardiovascular effects and molecular targets of resveratrol. Nitric Oxide 2012;26:102-10.

14. Price NL, Gomes A, Ling AY, et al. SIRT1 Is Required for AMPK Activation and the Beneficial Effects of Resveratrol on Mitochondrial Function. Cell Metabolism 2012;15:675-90.

15. Bellezza I, Giambanco I, Minelli A, et al. Nrf2-Keap1 signaling in oxidative and reductive stress. Biochim Biophys Acta Mol Cell Res 2018;1865:721-33.

16. Hu X, Rajesh M, Zhang J, et al. Protection by dimethyl fumarate against diabetic cardiomyopathy in type 1 diabetic mice likely via activation of nuclear factor 
erythroid-2 related factor 2. Toxicol Lett 2018;287:131-41.

17. Tan Y, Ichikawa T, Li J, et al. Diabetic downregulation of Nrf2 activity via ERK contributes to oxidative stressinduced insulin resistance in cardiac cells in vitro and in vivo. Diabetes 2011;60:625-33.

18. Miao Y, Wan Q, Liu X, et al. miR-503 Is Involved in the Protective Effect of Phase II Enzyme Inducer (CPDT) in Diabetic Cardiomyopathy via Nrf2/ARE Signaling Pathway. Biomed Res Int 2017;2017:9167450.

19. Huang K, Cheng C. Polydatin promotes Nrf2-ARE antioxidative pathway through activating Sirt1 to resist AGEsinduced upregulation of fibronetin and transforming growth factor- $\beta 1$ in rat glomerular messangial cells. Mol Cell Endocrinol 2015;399:178-89.

20. Deng C, Sun Z, Tong G, et al. $\alpha$-Lipoic acid reduces infarct size and preserves cardiac function in rat myocardial ischemia/reperfusion injury through activation of PI3K/ Akt/Nrf2 pathway. PLoS One 2013;8:e58371.

21. Waldman M, Cohen K, Yadin D, et al. Regulation of diabetic cardiomyopathy by caloric restriction is mediated by intracellular signaling pathways involving 'SIRT1 and PGC-1 $\alpha$ '. Cardiovasc Diabetol 2018;17:111.

22. Zhang Y, Babcock SA, Hu N, et al. Mitochondrial aldehyde dehydrogenase (ALDH2) protects against streptozotocininduced diabetic cardiomyopathy: role of GSK3 $\beta$ and mitochondrial function. BMC Med, 2012;10:40.

23. Yan Y, Tong F, Chen J. Endogenous BMP-4/ROS/COX2 mediated IPC and resveratrol alleviated brain damage. Curr Pharm Des 2019;25:1030-9.

24. Wang L, Zhang L, Chen ZB, et al. Icariin enchances neuronal survival after oxygen and glucose deprivation by increasing SIRT1. Eur J Pharmacol 2009;609:40-4.

25. Xiao Q, Ye Q, Wang W, et al. Mild hypothermia pretreatment protects against liver i-schemia reperfusion injury via the PI3K/AKT/FOXO3a pathway. Mol Med Rep 2017;16:7520-6.

26. King AJ. The use of animal models in diabetes research. Br J Pharmacol 2012;166:877-94.

27. Lochner A, Marais E, Genade S, et al. Protection of ischemia heart: investigations into the phenomenon of ischemia preconditioning. Cardiovasc J Afr 2009;20:43-51.

28. Schweiger S, Matthes F, Posey K, et al. Resveratrol induces dephosphorylation of Tau by interfering with the MID1PP2A complex. Sci Rep 2017;7:13753.

29. Wang X, Meng L, Zhao L, et al. Resveratrol ameliorates hyperglycemia-induced renal tubular oxidative stress damage via modulating the SIRT1/FOXO3a pathway. Diabetes Res Clin Pract 2017;126:172-81.
30. Shen C, Cheng W, Yu P, et al. Resveratrol pretreatment attenuates injury and promotes proliferation of neural stem cells following oxygen-glucose deprivation/reoxygenation by upregulating the expression of Nrf2, HO-1 and NQO1 in vitro. Mol Med Rep 2016;14:3646-54.

31. Cheng L, Jin Z, Zhao R, et al. Resveratrol attenuates inflammation and oxidative stress induced by myocardial ischemia-reperfusion injury: role of Nrf2/ARE pathway. Int J Clin Exp Med 2015;8:10420-8.

32. Peake BF, Nicholson CK, Lambert JP, et al. Hydrogen sulfide preconditions the $\mathrm{db} / \mathrm{db}$ diabetic mouse heart against ischemia-reperfusion injury by activating $\mathrm{Nrf2}$ signaling in an Erk-dependent manner. Am J Physiol Heart Circ Physiol 2013;304:H1215-24.

33. Wang YH, Li SA, Huang CH, et al. Sirt1 Activation by Post-ischemic Treatment With Lumbrokinase Protects Against Myocardial Ischemia-Reperfusion Injury. Front Pharmacol 2018;9:636.

34. Yu L, Li S, Tang X, et al. Diallyl trisulfide ameliorates myocardial ischemia-reperfusion injury by reducing oxidative stress and endoplasmic reticulum stress-mediated apoptosis in type 1 diabetic rats: role of SIRT1 activation. Apoptosis 2017;22:942-54.

35. Xue F, Huang JW, Ding PY, et al. Nrf2/antioxidant defense pathway is involved in the neuroprotective effects of Sirt1 against focal cerebral ischemia in rats after hyperbaric oxygen preconditioning. Behav Brain Res 2016;309:1-8.

36. Voelkl J, Mia S, Meissner A, et al. PKB/SGK-Resistant GSK-3 Signaling Following Unilateral Ureteral Obstruction. Kidney Blood Press Res 2013;38:156-64.

37. Juhaszova M, Zorov DB, Kim SH, et al. Glycogen synthase kinase- $3 \beta$ mediates convergence of protection signaling to inhibit the mitochondrial permeability transition pore. J Clin Invest 2004;113:1535-49.

38. Liu J, Li Y, Tang Y, et al. Rhein protects the myocardiac cells against hypoxia/reoxygention-induced injury by suppressing GSK3 $\beta$ activity. Phytomedicine 2018;51:1-6.

39. Kim KM, Lee KS, Lee GY, et al. Anti-diabetic efficacy of KICG1338, a novel glycogen synthase kinase- $3 \beta$ inhibitor, and its molecular characterization in animal models of type 2 diabetes and insulin resistance. Mol Cell Endocrinol 2015;409:1-10.

Cite this article as: $\mathrm{Xu} \mathrm{G}, \mathrm{Zhao} \mathrm{X}, \mathrm{Fu} \mathrm{J}$, Wang X. Resveratrol increase myocardial $\mathrm{Nrf} 2$ expression in type 2 diabetic rats and alleviate myocardial ischemia/reperfusion injury (MIRI). Ann Palliat Med 2019;8(5):565-575. doi: 10.21037/apm.2019.11.25 